\title{
Bernstein polynomials and spectral numbers for linear free divisors
}

\author{
Christian Sevenheck
}

February 19, 2019

\begin{abstract}
We discuss Bernstein polynomials of reductive linear free divisors. We define a suitable Brieskorn lattice for these non-isolated singularities, and show the analogue of Malgrange's result relating the roots of the Bernstein polynomial to the residue eigenvalues on the saturation of these Brieskorn lattices.
\end{abstract}

\section{Introduction}

In this note, we show that for reductive linear free divisors $D \subset \mathbb{C}^{n}$, which were studied in a number of recent papers (see [BM06] [GMNS09], MdGS08] and [GS08]), the roots of the Bernstein polynomial of a defining equation $h$ of $D$ can be recovered as a certain set of eigenvalues of a residue endomorphism. More precisely, for a generic linear form $f$ on $\mathbb{C}^{n}$, one defines a family of Gauß-Manin systems for $f$, seen as a function on the fibres of $h$. This family has a specific (logarithmic) extension over $D$, which gives the set of residue eigenvalues we are interested in.

This relation to the Bernstein polynomial has a number of consequences: First, while the definition of the Bernstein polynomial is rather simple, it is in general very hard to calculate its roots in concrete examples. This is true even for linear free divisors, though the differential operator occurring in Bernstein's functional equation is more explicitly known than in the general case. On the other hand, the calculation of the residue eigenvalues alluded to above is, although not trivial, easier to carry out. We apply our result to obtain, using the calculations from [MdGS08, chapter 6], Bernstein polynomials for discriminants in representation spaces of the Dynkin quivers $A_{n}, D_{n}$ and $E_{6}$ as well as the so-called star quiver $\star_{n}$, also considered in loc.cit. (which is not a Dynkin quiver for $n>3$ ).

Another motivation for this work comes from the fact that the residue eigenvalues of the Gauß-Manin systems give information on their limit behavior (resp., of the corresponding family of Brieskorn lattices), when approaching the zero fibre of $h$, i.e., the divisor $D$. In particular, in MdGS08], questions about the degeneration of Frobenius manifolds, associated to the tame functions $f_{\mid D_{t}}$, where $D_{t}:=h^{-1}(t)$, were related to the asymptotic behavior of a natural pairing defined on the Gauß-Manin system. In particular, the residue eigenvalues of these Gauß-Manin systems then need to be symmetric around zero. This was stated as a conjecture in loc.cit., and it follows from the relation between these eigenvalues and the roots of the Bernstein polynomial that we prove here.

Finally, the family of Brieskorn lattices associated to $f_{\mid D_{t}}$ also has logarithmic extension over the divisor $D$, constructed using logarithmic differential forms. We define the fibre over $t=0$ to be the logarithmic Brieskorn lattice of $D$ (in fact, it does not depend on the choice of the linear form). In contrast to the fibres at $t \neq 0$, this Brieskorn lattice is regular singular at the origin, reflecting the local situation of the pair $(f, h)$ at the origin in $\mathbb{C}^{n}$. It turns out that then our result can be rephrased to give the analogue of Malgrange's classical result for isolated singularities: The roots of the Bernstein polynomial are (up to a rescaling) the residue eigenvalues on the saturation of this logarithmic Brieskorn lattice.

2000 Mathematics Subject Classification. 32S40, 34M35.

Keywords: Brieskorn lattice, Bernstein polynomial, linear free divisors, spectral numbers.

This research is partially supported by ANR grant ANR-08-BLAN-0317-01 (SEDIGA). 
Acknowledgements: I thank Claude Sabbah and Michel Granger for their help during the preparation of this article. I am particularly grateful to Ignacio de Gregorio for all the discussions on linear free divisors and related subjects we had over the last years and also for having done a good part of the computations used in this paper.

\section{Linear Free Divisors and Gauß-Manin systems}

In this section, we first recall from MdGS08 the construction of the family of Gauß-Manin systems associated to a linear section of a linear free divisor. We also give a more intrinsic definition of these Gauß-Manin systems as a direct image of a map constructed from two polynomials. Finally, we discuss the definition of the residue eigenvalues relevant for the present work, as introduced in MdGS08.

We denote throughout this article by $V$ the complex vector space $\mathbb{C}^{n}$.

Definition-Lemma 1 (Sai80, [BM06], [MdGS08]). 1. Let $D \subset V$ be a reduced hypersurface with defining equation $h$. Then $D$ is called a free divisor, if the sheaf $\Theta_{V}(-\log D):=\left\{\vartheta \in \Theta_{V} \mid \vartheta(h) \subset\right.$ $(h)\}$ is a free $\mathcal{O}_{V}$-module. If moreover a basis $\left(\xi_{i}\right)$ of $\Theta_{V}(-\log D)$ exists such that $\xi_{i}=\sum_{j=1}^{n} \xi_{i j} \partial_{x_{j}}$ where $\xi_{i j}$ are linear forms on $V$, then $D$ is called linear free.

2. Let $G$ be the identity component of the algebraic group $G_{D}:=\{g \in \mathrm{Gl}(V) \mid g(D) \subset D\}$. Then $(V, G)$ is a pre-homogenous vector space in the sense of Sato (see, e.g., SKYך), in particular, the complement $V \backslash D$ is an open orbit of $G$. We call $D$ reductive if $G_{D}$ is so. A rational function $r \in \mathbb{C}(V)$ is called a semi-invariant if there is a character $\chi_{r}: G \rightarrow \mathbb{C}^{*}$ such that $g(r)=\chi_{r}(g) \cdot r$ for all $g \in G$. Obviously, $h$ itself is a semi-invaraint.

3. $G$ acts on $V^{*}$ by the dual action, with dual discriminant $D^{*} \subset V^{*}$. If $G_{D}$ is reductive, then $\left(V^{*}, D^{*}\right)$ is pre-homogenous. We call a linear form $f \in V^{*}$ generic with respect to $h$ (or simply generic, if no confusion is possible) if $f$ lies in the open orbit $V^{*} \backslash D^{*}$ of the dual action.

There is a basis $\left(e_{i}\right)$ of $V$ with corresponding coordinates $\left(x_{i}\right)$ (called unitary) such that $G$ appears as a subgroup of $U(n)$ in these coordinates. Then $D^{*}=\left\{h^{*}=0\right\}$, where $h^{*}(y):=\overline{h(\bar{x})},\left(y_{i}\right)$ being the dual coordinates of $\left(x_{i}\right)$.

In the sequel, we always consider linear forms which are generic with respect to $h$.

In order to study the behavior of the restriction of the linear function $f$ on the fibres $D_{t}:=h^{-1}(t), t \neq 0$, but also on $D$ itself, the following deformation algebra was introduced in MdGS08.

Definition 2. Let $D$ be linear free.

1. Let $E \in \Theta_{V}(-\log D)$ be the Euler field $E=\sum_{i=1}^{n} x_{i} \partial_{x_{i}}$. Call

$$
\Theta_{V / T}(-\log D):=\left\{\vartheta \in \Theta_{V}(-\log D) \mid \vartheta(h)=0\right\}
$$

the module of relative logarithmic vector fields. $\Theta_{V / T}(-\log D)$ is $\mathcal{O}_{V}$-free of rank $n-1$, and we have a decomposition $\Theta_{V}(-\log D)=\mathcal{O}_{V} E \oplus \Theta_{V / T}(-\log D)$.

2. The ideal $J_{h}(f):=d f\left(\Theta_{V / T}(-\log D)\right) \subset \mathcal{O}_{V}$ is called the Jacobian ideal of the pair $(f, h)$. The quotient $\mathcal{O}_{V} / J_{h}(f)$ is the Jacobian algebra (or deformation algebra) of $(f, h)$.

Notice that $\Theta_{V / T}(-\log D)$ was called $\Theta_{V}(-\log h)$ in MdGS08. It was shown in loc.cit., section 3.2, that if $f$ is generic with respect to $h$, then $h_{*} \mathcal{O}_{V} / J_{h}(f)$ is $\mathcal{O}_{T}$-free of rank $n$, and generated by $\left(f^{i}\right)$ for $i=0, \ldots, n-1$. Moreover, it is interpreted as the relative tangent space $T_{\mathcal{R}_{h} / \mathbb{C}}^{1}(f)$ of the deformation theory of $f$ with respect to the group $\mathcal{R}_{h}$ of right-equivalences preserving all fibres of $h$.

Denote by $\left(\Omega_{V / T}^{\bullet}(\log D), d\right):=\left(\Omega_{V}^{\bullet}(\log D) /\left(\Omega_{V}^{\bullet-1}(\log D) \wedge h^{*} \Omega^{1}(\log \{0\}), d\right)\right.$ the relative logarithmic de Rham complex of $h$ as studied, under the name $\Omega^{\bullet}(\log h)$ in MdGS08, section 2.2]). This relative logarithmic complex is used in the definition of the family of Gauß-Manin-systems resp. Brieskorn lattices in loc.cit. 
Definition-Lemma 3 (MdGS08, section 4]). Let $h$ and $f$ as above. Define

$$
\begin{aligned}
& G(\log D) \quad:=\frac{H^{0}\left(V, \Omega_{V / T}^{n-1}(\log D)\left[\theta, \theta^{-1}\right]\right)}{(\theta d-d f \wedge) H^{0}\left(V, \Omega_{V / T}^{n-2}(\log D)\left[\theta, \theta^{-1}\right]\right)} \quad ; \quad G(* D) \quad:=\frac{H^{0}\left(V, \Omega_{V / T}^{n-1}(* D)\left[\theta, \theta^{-1}\right]\right)}{(\theta d-d f \wedge) H^{0}\left(V, \Omega_{V / T}^{n-2}(* D)\left[\theta, \theta^{-1}\right]\right)} \\
& G_{0}(\log D):=\frac{H^{0}\left(V, \Omega_{V / T}^{n-1}(\log D)[\theta]\right)}{(\theta d-d f \wedge) H^{0}\left(V, \Omega_{V / T}^{n-2}(\log D)[\theta]\right)} \quad ; \quad G_{0}(* D) \quad:=\frac{H^{0}\left(V, \Omega_{V / T}^{n-1}(* D)[\theta]\right)}{(\theta d-d f \wedge) H^{0}\left(V, \Omega_{V / T}^{n-2}(* D)[\theta]\right)} .
\end{aligned}
$$

Then $G(* D)$ is $\mathbb{C}\left[\theta, \theta^{-1}, t, t^{-1}\right]$-free of rank $n$ and $G(\log D)\left(\right.$ resp. $\left.G_{0}(* D), G_{0}(\log D)\right)$ is a $\mathbb{C}\left[\theta, \theta^{-1}, t\right]$ (resp. $\mathbb{C}\left[\theta, t, t^{-1}\right]-, \mathbb{C}[\theta, t]$-) lattice inside $G(* D)$. These modules fit into the following diagram

$$
\begin{array}{ccc}
G(\log D) & \subset & G(* D) \\
\cup & & \cup \\
G_{0}(\log D) & \subset & G_{0}(* D) .
\end{array}
$$

Define a connection

$$
\nabla: G_{0}(\log D) \longrightarrow G_{0}(\log D) \otimes \theta^{-1} \Omega_{\mathbb{C} \times T}^{1}(\log (\{0\} \times T) \cup(\mathbb{C} \times\{0\}))
$$

by putting, for a form $\omega \in H^{0}\left(V, \Omega_{V / T}^{n-1}(\log D)\right)$,

$$
\begin{aligned}
& \nabla_{\partial_{\theta}}([\omega]):=\theta^{-2}[f \cdot \omega] \\
& \nabla_{\partial_{t}}([\omega]):=\frac{1}{n t}\left(\left[\operatorname{Lie}_{E}(\omega)\right]-\left[\theta^{-1} f \cdot \omega\right]\right)
\end{aligned}
$$

and extending by the Leibniz-rule (for $\nabla_{\partial_{\theta}}$ ) resp. $\theta$-linearly (for $\nabla_{\partial_{t}}$ ). We denote by $\nabla$ the induced connection on $G(\log D), G_{0}(* D)$ and $G(* D)$.

One of the main results of MdGS08] concerns the construction of various bases of the module $G_{0}(\log D)$ (hence, of all the other modules given above), such that the connection takes a particularly simple form. This can be summarized as follows.

Proposition 4 (MdGS08, proposition 4.5(iii)]). There is a $\mathbb{C}[\theta, t]$-basis $\underline{\omega}^{(1)}=\left(\omega_{1}, \ldots, \omega_{n}\right)$ of $G_{0}(\log D)$ such that

$$
\nabla\left(\underline{\omega}^{(1)}\right)=\underline{\omega}^{(1)} \cdot\left[\left(A_{0} \frac{1}{\theta}+A_{\infty}\right) \frac{d \theta}{\theta}+\left(-A_{0} \frac{1}{\theta}+A_{\infty}^{\prime}\right) \frac{d t}{n t}\right]
$$

where

$$
A_{0}:=\left(\begin{array}{ccccc}
0 & 0 & \ldots & 0 & c \cdot t \\
-1 & 0 & \ldots & 0 & 0 \\
\vdots & \vdots & \ddots & \vdots & \vdots \\
0 & 0 & \ldots & 0 & 0 \\
0 & 0 & \ldots & -1 & 0
\end{array}\right),
$$

$A_{\infty}=\operatorname{diag}\left(\nu_{1}, \ldots, \nu_{n}\right)$ and $A_{\infty}^{\prime}:=\operatorname{diag}(0,1, \ldots, n-1)-A_{\infty}$. The constant $c \in \mathbb{C}$ is defined by the equation

$$
f^{n}=-c \cdot h+\sum_{i=1}^{n-1} \xi_{i}(f) \cdot k_{i},
$$

where $\xi_{i} \in \Theta_{V / T}(\log D)$ and $k_{i} \in \mathcal{O}_{V}$ are homogenous polynomials of degree $n-1$. Here

$$
\omega_{1}=\iota_{E} \frac{\mathrm{vol}}{h}=n \frac{\mathrm{vol}}{d h}
$$

where $\mathrm{vol}=d x_{1} \wedge \ldots \wedge d x_{n}$. In particular, $(G(* D), \nabla)$ is flat. Moreover, $\left(\nu_{1}, \ldots, \nu_{n}\right)$ is the spectrum at $(\theta=)$ infinity of the restriction of $G_{0}(\log D)$ to $t=0$.

The following obvious consequence will be used in the next section. 
Corollary 5. Consider the inclusion $j:\{1\} \times T \hookrightarrow \mathbb{C} \times T$ and the restriction $\left(G_{1}(* D), \nabla\right):=$ $j^{*}(G(* D), \nabla)$. This is a meromorphic bundle on $T$ with connection (the only pole being at $0 \in T$ ), and can thus be seen as a coherent and holonomic left $\mathbb{C}[t]\left\langle\partial_{t}\right\rangle$-module. Then we have an isomorphism of left $\mathbb{C}[t]\left\langle\partial_{t}\right\rangle$-modules

$$
\varphi: \mathbb{C}[t]\left\langle\partial_{t}\right\rangle /\left(b_{G_{1}(\log D)}\left(t \partial_{t}\right)-c \cdot t\right) \stackrel{\cong}{\longrightarrow}\left(G_{1}(* D), \nabla\right),
$$

where $b_{G_{1}(\log D)}$ is the spectral polynomial of $G_{1}(\log D):=j^{*}(G(\log D), \nabla)$ at $0 \in T$, i.e.,

$$
b_{G_{1}(\log D)}(s):=\prod_{i=1}^{n}\left(\frac{i-\nu_{i}}{n}-s\right),
$$

and where $\varphi(1)=\omega_{1}$. In particular, $\omega_{1}$ satisfies the functional equation $b_{G_{1}(\log D)}\left(t \partial_{t}\right) \omega_{1}=c \cdot t \omega_{1}$ in $G_{1}(* D)$.

We note the following easy consequence from the definitions, which is not discussed in MdGS08.

Lemma 6. Let $f_{1}, f_{2} \in V^{*} \backslash D^{*}$ be two generic linear forms. Denote by $\left(G_{i}(\log D), \nabla_{i}\right)$ the family of Brieskorn lattices attached to the pair $\left(f_{i}, h\right), i=1,2$. Then $\varphi_{c^{\prime}}^{*}\left(G_{1}(\log D), \nabla_{1}\right) \cong\left(G_{2}(\log D), \nabla_{2}\right)$, where $\varphi_{c^{\prime}}: \mathbb{C} \times T \rightarrow \mathbb{C} \times T$ is defined as $\varphi(\theta, t)=\left(\theta, c^{\prime} \cdot t\right)$ for some $c^{\prime} \in \mathbb{C}^{*}$.

Proof. By definition, the complement of $D^{*}$ in $V^{*}$ is an (open) orbit of the dual action of $G$, hence, there is $g \in G$ with $g\left(f_{1}\right)=f_{2}$. Then $g(h)=\chi_{h}(g) \cdot h$ and it follows that $\varphi_{\chi_{h}(g)}^{*}\left(G_{1}(\log D), \nabla_{1}\right) \cong$ $\left(G_{2}(\log D), \nabla_{2}\right)$.

In order to relate the above objects to the Bernstein polynomial of $h$, we recall how the Gauß-Manin system $G(* D)$, seen as a left $\mathbb{C}[\theta, t]\left\langle\partial_{\theta}, \partial_{t}\right\rangle$-module is obtained as a direct image of a differential system on $V$. Notice that the following lemma is merely a variant of [DS03, proposition 2.7 and theorem 2.9]. We consider $f$ as a morphism $f: V \rightarrow R=\operatorname{Spec} \mathbb{C}[r]$, and put $\Phi:=(f, h): V \rightarrow R \times T$.

Lemma 7. Let $\Phi_{+} \mathcal{O}_{V}(* D)$ be the (algebraic) direct image complex of the holonomic $\mathcal{D}_{V}$-module $\mathcal{O}_{V}(* D)$. Then

1. The cohomology sheaves of $\Phi_{+} \mathcal{O}_{V}(* D)$ are $\mathcal{D}_{R \times T}$-coherent, holonomic and regular.

2. $\Phi_{+} \mathcal{O}_{V}(* D)$, seen in $\mathcal{D}^{b}\left(\mathcal{D}_{R \times T / T}\right)$ is represented by

$$
\left(\Phi_{*} \Omega_{V / T}^{\bullet-1}(* D)\left[\partial_{r}\right], d-\left(d f \wedge-\otimes \partial_{r}\right)\right) .
$$

Under the isomorphism

$$
\mathcal{H}^{0}\left(\Phi_{+} \mathcal{O}(* D)\right) \cong \frac{\Phi_{*} \Omega_{V / T}^{n-1}(* D)\left[\partial_{r}\right]}{\left(d-d f \wedge-\otimes \partial_{r}\right) \Phi_{*} \Omega_{V / T}^{n-2}(* D)\left[\partial_{r}\right]}
$$

the action of $\partial_{t}$ on a class $[\omega] \in \mathcal{H}^{0}\left(\Phi_{+} \mathcal{O}_{V}(* D)\right)$ represented by a form $\omega \in \mathcal{H}_{V / T}^{n-1}(* D)$ is given by

$$
\partial_{t}([\omega]):=\frac{1}{n \cdot t}\left(\left[\operatorname{Lie}_{E}(\omega)\right]-\left[\operatorname{Lie}_{E}(f) \omega \otimes \partial_{r}\right]\right)
$$

3. Put $\left.M:=\mathbb{H}^{0}\left(R \times T, \Phi_{+} \mathcal{O}(* D)\right)\right)$. Denote by $\widehat{M}$ the partial Fourier-Laplace transformation with respect to $r$ of $M$, i.e., $\widehat{M}=M$ as $\mathbb{C}$-vector spaces, and we define an structure of a $\mathbb{C}[\tau, t]\left\langle\partial_{\tau}, \partial_{t}\right\rangle$ module on $\widehat{M}$ by $\tau \cdot:=\partial_{r}$ and $\partial_{\tau}:=-r$. . Then $\widehat{M}$ is $\mathbb{C}[\tau, t]\left\langle\partial_{\tau}, \partial_{t}\right\rangle$-holonomic, with singularities at $\tau=\{0, \infty\}$ and $t=0$ at most, the one at $\tau=0$ and $\{t=0, \tau \neq \infty\}$ being regular (but not the one at $\tau=\infty$ in general). Moreover, by putting $\theta=\tau^{-1}$, the localized Fourier-Laplace transformation $\widehat{M}\left[\tau^{-1}\right]$ of $M$ is isomorphic to $G(* D)$ as a meromorphic vector bundle with connection (the one on $G(* D)$ being given by formula (2)). 
4. The restriction $\left(G_{1}(* D), \nabla\right)$ is isomorphic (as a left $\mathbb{C}[t]\left\langle\partial_{t}\right\rangle$-module) to $\mathbb{H}^{0}\left(T, h_{+} \mathcal{O}_{V}(* D) e^{-f}\right)$, where $\mathcal{O}_{V}(* D) e^{-f}$ is the tensor product of $\mathcal{O}_{V}(* D)$ with a rank one $\mathcal{O}_{V}$-module formally generated by $e^{-f}$, i.e., $\mathcal{O}_{V}(* D) e^{-f} \cong \mathcal{O}_{V}(* D)$ as $\mathcal{O}_{V}$-modules, and the differential of $\mathcal{O}_{V}(* D) e^{-f}$ (i.e., the operator defining its $\mathcal{D}_{V}$-module structure) is given by $d_{f}:=d-d f \wedge$.

Proof. 1. see [Meb04, theorème 9.0-8.]

2. It is well known that for any left $\mathcal{D}_{V}$-module $\mathcal{M}$, the direct image complex $\Phi_{+} \mathcal{M}$ is represented by

$$
\left(\mathbb{R} \Phi_{*} \Omega_{V}^{n+\bullet}(\mathcal{M})\left[\partial_{r}, \partial_{t}\right], d-\left(d f \wedge-\otimes \partial_{r}\right)-\left(d h \wedge-\otimes \partial_{t}\right)\right) .
$$

Putting $\mathcal{M}=\mathcal{O}_{V}(* D)$ and using that $\Phi$ is affine, we consider the double complex given by

$$
E^{p, q}:=\left(\left(\Phi_{*} \Omega_{V}^{p+q}(* D)\left[\partial_{r}\right]\right) \otimes \partial_{t}^{q}, d-\left(d f \wedge-\otimes \partial_{r}\right),-\left(d h \wedge-\otimes \partial_{t}\right)\right)
$$

which abuts to $\mathcal{H}^{p+q-n}\left(\Phi_{+}\left(\mathcal{O}_{V}(* D)\right)\right)$. The morphism $h$ is smooth restricted to $V \backslash D$, hence, the Koszul complex $\left(\Omega^{\bullet}(* D), d h \wedge\right)$ is acyclic. Therefore the second spectral sequence associated to the above double complex degenerates at the $E_{2}$-term and the isomorphism

$$
\Omega_{V / T}^{p-1}(* D) \stackrel{d h \wedge}{\longrightarrow} \operatorname{Ker}\left(\Omega_{V}^{p}(* D) \stackrel{d h \wedge}{\longrightarrow} \Omega_{V}^{p+1}(* D)\right)
$$

yields the above quasi-isomorphism.

In order to prove the formula for the action of $\partial_{t}$, notice that given a class $[\omega]$ defined by a relative $n$-form $\omega \in \Omega_{V / T}^{n}(* D)$, the class corresponding to it in $\mathcal{H}^{0}\left(\Phi_{+} \mathcal{O}_{V}(* D)\right)$ is $[d h \wedge \omega]$. By definition, we have $\partial_{t}([d h \wedge \omega])=\left[d h \wedge \omega \otimes \partial_{t}\right]$. This class is equal in $\mathcal{H}^{0}\left(\Phi_{+} \mathcal{O}_{V}(* D)\right)$ to $\left[d \omega-d f \wedge \omega \otimes \partial_{r}\right]$. It follows that under the isomorphism (雨), this equals $\left[\frac{d \omega}{d h}-\frac{d f \wedge \omega}{d h} \otimes \partial_{r}\right] \in \Phi_{*} \Omega_{V / T}^{n-1}(* D)\left[\partial_{r}\right] /(d-d f \wedge$ $\left.-\otimes \partial_{r}\right) \Phi_{*} \Omega_{V / T}^{n-2}(* D)$. Now notice that as $h$ is smooth outside $D$, there is a vector field $X \in \Theta_{V}(* D)$ which lifts $\partial_{t} \in \Theta_{T}$. Then we have that $d \omega / d h=\iota_{X} d \omega$ and $(d f \wedge \omega) / d h=\iota_{X}(d f) \wedge \omega-d f \wedge \iota_{X} \omega$. Putting this together and using once again the relation in the quotient $\Phi_{*} \Omega_{V / T}^{n-1}(* D)\left[\partial_{r}\right] /(d-d f \wedge$ $\left.-\otimes \partial_{r}\right) \Phi_{*} \Omega_{V / T}^{n-2}(* D)$ one arrives at the formula

$$
\partial_{t}[\omega]=\left[\operatorname{Lie}_{X} \omega\right]-[\operatorname{Lie}(f) \omega] \otimes \partial_{r} .
$$

Now the result follows as the meromorphic vector field $X$ is simply $E /(n \cdot h)$, due to the homogeneity of $h$.

3. This is obvious from the last point: Fourier-Laplace transformation and localization along $\tau=0$ transforms formula (14) into the defining equation (11) of $G(* D)$ and formula (5) obviously corresponds to the second part of formula (2). The statements about regularity follows form the general considerations in [DS03, theorem 1.11], using the regularity of $\Phi_{+} \mathcal{O}_{V}(* D)$ from 1.

4. By definition, $h_{+} \mathcal{O}_{V}(* D) e^{-f}$ is represented by the complex

$$
\left(h_{*} \Omega_{V}^{n+\bullet}(* D)\left[\partial_{t}\right], d-d f \wedge-\left(d h \wedge-\otimes \partial_{t}\right)\right) .
$$

The same argument as above shows that this is quasi-isomorphic to

$$
\left(h_{*} \Omega_{V / T}^{n-1+\bullet}(* D), d-d f \wedge\right) .
$$

Now it is clear that

$$
\mathbb{H}^{0}\left(T, h_{*} \Omega_{V / T}^{n-1+\bullet}(* D), d-d f \wedge\right)=\frac{H^{0}\left(\Omega_{V / T}^{n-1}(* D)\right)}{\left.(d-d f \wedge) H^{0}\left(V, \Omega_{V / T}^{n-2}(* D)\right)\right)}=G_{1}(* D)
$$

Remark: Direct images of regular holonomic modules by a morphism consisting of two polynomials occur in the work of C. Roucairol (see Rou06a, Rou07 and Rou06b). She also studied direct images of twisted modules, i.e., $h_{+}\left(\mathcal{M} e^{-f}\right)$. However, we will not need her results directly as the computations from MdGS08] (i.e. proposition 1 above) give already very precise information about these direct images for a pair $(f, h)$, with $h$ reductive linear free and $f$ linear and generic. 


\section{Bernstein Polynomials}

We first give the definition of the Bernstein polynomial through the classical functional equation. Next we recall how this can be rephrased using the general theory of $V$-filtrations. This will be useful in proving the main result. Finally, we state and prove the relation between the roots of the Bernstein polynomial of a defining equation $h$ for a linear free divisor and the residue eigenvalues of the family of Gauß-Manin-systems introduced in section 2.

The following classical statement is due to Bernstein (see, Ber72]).

Theorem 8. Let $h \in \mathcal{O}_{V}$ be any function, then there is a polynomial $B \in \mathbb{C}[s]$ and a differential operator $P\left(x_{i}, \partial_{x_{i}}, s\right) \in \mathcal{D}_{V}[s]$ such that

$$
P\left(x_{i}, \partial_{x_{i}}, s\right) h^{s+1}=B(s) h^{s}
$$

All polynomials $B(s) \in \mathbb{C}[s]$ having this property form an ideal in $\mathbb{C}[s]$, and we denote by $b_{h}(s)$ the unitary generator of this ideal. $b_{h}(s)$ is called the Bernstein polynomial of $h$.

If $h$ defines a linear free divisor, then the theory of pre-homogenous vector spaces shows that the functional equation defining $b_{h}(s)$ is of a particular type.

Theorem 9 ([SK77], Gyo91, GS08]). Let $D=h^{-1}(0)$ be a reductive linear free divisor, then the operator $P$ appearing in Bernstein's functional equation is given by $P:=h^{*}\left(\partial_{x_{1}}, \ldots, \partial_{x_{1}}\right)$ (remember that $h^{*}(\underline{y})=\overline{h(\underline{\bar{x}})}$, where $x_{i}$ are the unitary coordinates and $y_{i}$ are their duals). In particular, it is an element of $\mathbb{C}\left\langle\partial_{x_{1}}, \ldots, \partial_{x_{n}}\right\rangle$. Moreover, the degree of $b_{h}(s)$ is equal to $n$ and the roots of $b_{h}(s)$ are contained in the open intervall $(-2,0)$ and are symmetric around -1 . In particular, -1 is the only integer root.

The following classical reformulation of the definition of the Bernstein polynomial will be useful in the sequel.

Consider the ring $\mathbb{C}\left[s, h^{-1}\right]$, and denote by $M\left[h^{-1}\right]:=\mathbb{C}\left[s, h^{-1}\right] h^{s+1}$ the rank one $\mathbb{C}\left[s, h^{-1}\right]$-module generated by the symbol $h^{s+1}$. Define an action of $\mathcal{D}_{V}$, the ring of algebraic differential operators on $V$ on $M\left[h^{-1}\right]$ by putting $\partial_{x_{i}}\left(g \cdot h^{s+1}\right):=\partial_{x_{i}}(g) \cdot h^{s+1}+g \cdot(s+1) h^{-1} \partial_{x_{i}}(h) h^{s+1}$. This action extends naturally to an $\mathcal{D}_{V}[s]$-action. Let $M$ the $\mathcal{D}_{V}[s]$-submodule of $M\left[h^{-1}\right]$ generated by $h^{s+1}$. Define an action of $t$ on $M\left[h^{-1}\right]$ by putting $t\left(g(s) \cdot h^{s+1}\right):=g(s+1) \cdot h \cdot h^{s+1}$. Then $b_{h}(s)$ is the minimal polynomial of the action of $s$ on the quotient $M / t M$.

This definition can be rephrased once more using the theory of $V$-filtrations on $\mathcal{D}$-modules. Without reviewing the details of the theory, we recall the following facts (see, e.g. [MM04, section 4])

Definition-Lemma 10. Let $X$ be any smooth algebraic variety, and $Y \subset X$ a smooth hypersurface defined by an ideal sheaf $I \subset \mathcal{O}_{X}$. We denote by $t \in \mathcal{O}_{X}$ a local generator of $\mathcal{I}$.

1. Let $\mathcal{D}_{X}$ be the sheaf of algebraic differential operators, then define

$$
V_{k} \mathcal{D}_{X}:=\left\{P \in \mathcal{D}_{X} \mid P\left(I^{j}\right) \subset I^{j-k}\right\}
$$

For any left $\mathcal{D}_{X}$-module $\mathcal{M}$, a $V$-filtration on $\mathcal{M}$ is an increasing filtration $U \cdot \mathcal{M}$ compatible with $V_{\bullet} \mathcal{D}_{X}$.

2. A V-filtration $U \cdot \mathcal{M}$ on a left $\mathcal{D}_{X}$-module $\mathcal{M}$ is good iff the Rees-module $\oplus z^{k} U_{k} \mathcal{M}$ is $\mathcal{R}_{V} \mathcal{D}_{X}$ coherent, where $\mathcal{R}_{V} \mathcal{D}_{X}:=\oplus_{k} z^{k} V_{k} \mathcal{D}_{X}$.

3. A good V-filtration $U \cdot \mathcal{M}$ is said to have a Bernstein polynomial iff there is a non-zero polynomial $b(s) \in \mathbb{C}[s]$ such that for all $k \in \mathbb{Z}$, we have $b\left(t \partial_{t}+k\right) U_{k} \mathcal{M} \subset U_{k-1} \mathcal{M}$.

4. A coherent $\mathcal{D}_{X}$-module $\mathcal{M}$ is called specializable iff locally there exists a good $V$-filtration $U$. $\mathcal{M}$ having a Bernstein polynomial. Equivalently, for any local section $m \in \mathcal{M}$ there is a non-zero polynomial $b_{m}(s)$ (the Bernstein polynomial of $m$ ) such that $b_{m}\left(t \partial_{t}\right) m \in V_{-1} \mathcal{D}_{X} \cdot m$.

5. A holonomic $\mathcal{D}_{X}$-module is specializable along any smooth hypersurface $Y$.

The following evident corollary gives an example of a $V$-filtration that will be used later. 
Corollary 11. Consider the left $\mathbb{C}[t]\left\langle\partial_{t}\right\rangle$-module $G_{1}(* D)$ from above. Then

$$
U_{k} G_{1}(* D):=V_{k} \mathbb{C}[t]\left\langle\partial_{t}\right\rangle \cdot G_{1}(\log D)
$$

defines a good V-filtration on $G_{1}(* D)$, whose Bernstein polynomial is exactly $b_{G_{1}(\log D)}(s)$. Moreover, we have

$$
U_{0} G_{1}(* D)=G_{1}(\log D)=V_{0} \mathbb{C}[t]\left\langle\partial_{t}\right\rangle \cdot \omega_{1}
$$

We will also use $V$-filtrations for $\mathcal{D}_{T \times V}$-modules. The following result is well known, see, e.g., Mal75, [MM04, lemme 4.4-1].

Lemma 12. 1. Let $h \in \mathcal{O}_{V}$ an arbitrary function, seen as a morphism $h: V \rightarrow T$. Denote by $i_{h}: V \hookrightarrow T \times V$ the graph embedding, with image $\Gamma_{h}$. Put $\mathcal{N}:=\left(i_{h}\right)_{+} \mathcal{O}_{V}$, then $\mathcal{N} \cong \mathcal{O}_{V}\left[\partial_{t}\right] \cong$ $\mathcal{O}_{T \times V}\left(* \Gamma_{h}\right) / \mathcal{O}_{T \times V} \cong \mathcal{D}_{T \times V} \delta(t-h)$. A good $V$-filtration on $\mathcal{N}$ is defined by putting, for all $k \geq 0$, $U_{k} \mathcal{N}:=V_{k} \mathcal{D}_{T \times V} \delta(t-h)$, and for all $k<0, U_{k} \mathcal{N}:=t^{k} V_{0} \mathcal{D}_{T \times V} \delta(t-h)$. This $V$-filtration admits a Bernstein polynomial (namely, a Bernstein polynomial for the section $\delta(t-h)$ ), which is exactly the polynomial $b_{h}(-s-1)$. We denote, as in [Mal75], by $\mathcal{M}$ the $V_{0} \mathcal{D}_{T \times V}$-module $U_{0} \mathcal{N}$.

2. The direct image $\left(i_{h}\right)_{+} \mathcal{O}_{V}(* D)$ is the localization of both $\mathcal{N}$ and $\mathcal{M}$ along $t=0$, and is thus denoted by $\mathcal{M}\left[t^{-1}\right]$. As $\mathcal{N}$ has no t-torsion, we have an exact sequence

$$
0 \longrightarrow \mathcal{N} \longrightarrow \mathcal{M}\left[t^{-1}\right] \longrightarrow C \longrightarrow 0
$$

where $C$ is a $\mathcal{D}_{T \times V}$-module. A Bernstein polynomial for a local section $m \in \mathcal{N}$ is also a Bernstein polynomial for $m$, seen as a local section in $\mathcal{M}\left[t^{-1}\right]$.

We can now state and prove the main result of this paper.

Theorem 13. Let $D=h^{-1}(0)$ be reductive linear free divisor and $f \in V^{*}$ be generic. Consider the family of Gauß-Manin systems $G(* D)$, the logarithmic extension $G(\log D)$ and the restrictions $G_{1}(\log D) \subset$ $G_{1}(* D)$ from above. Then we have that $b_{h}(s)=b_{G_{1}(\log D)}(s+1)$ (recall that $b_{G_{1}(\log D)}(s)$ is the spectral polynomial of $\left.G_{1}(\log D)\right)$.

In order to prove this result, we start with a preliminary lemma.

Lemma 14. Let $\mathcal{M}\left[t^{-1}\right]:=\left(i_{h}\right)_{+} \mathcal{O}_{V}(* D)$ as above. Consider the twisted module $\left(i_{h}\right)_{+} \mathcal{O}_{V}(* D) e^{-f}$. Then the section $\delta(t-h) e^{-f} \in\left(i_{h}\right)_{+} \mathcal{O}_{V}(* D) e^{-f}$ admits $b_{h}(s)$ as a Bernstein polynomial, with associated functional equation

$$
\left(t \cdot h^{*}\left(\partial_{x_{i}}+a_{i}\right)-b_{h}\left(-\partial_{t} t\right)\right) \delta(t-h) e^{-f}=0
$$

where $f=\sum_{i=1}^{n} a_{i} x_{i}$

Proof. By lemma 12, $b_{h}(s)$ is the minimal polynomial of $-\partial_{t} t$ on $\frac{\mathcal{D}_{V}\left[t \partial_{t}\right] \delta(t-h)}{t \mathcal{D}_{V}\left[t \partial_{t}\right] \delta(t-h)}$. In particular, by theorem 8, the functional equation

$$
\left(t \cdot h^{*}\left(\partial_{x_{i}}\right)-b_{h}\left(-\partial_{t} t\right)\right) \delta(t-h)=0
$$

holds in $\left(i_{h}\right)_{+} \mathcal{O}_{V}(* D)$. Then it follows directly from the definition of the twisted module $\mathcal{O}_{V}(* D) e^{-f}$ that the functional equation (6) from above holds in $\left(i_{h}\right)_{+} \mathcal{O}_{V}(* D) e^{-f}$. Now suppose that there is another equation

$$
\left(t \cdot \widetilde{P}\left(x_{i}, \partial_{x_{i}},-\partial_{t} t\right)-\widetilde{B}\left(-\partial_{t} t\right)\right) \delta(t-h) e^{-f}=0
$$

where $\widetilde{P} \in \mathcal{D}_{V}[s]$ and $\widetilde{B}(s) \in \mathbb{C}[s]$ with $\operatorname{deg}(\widetilde{B})<\operatorname{deg}\left(b_{h}\right)$. Then we obtain the equation

$$
\left(t \cdot \widetilde{P}\left(x_{i}, \partial_{x_{i}}-a_{i},-\partial_{t} t\right)-\widetilde{B}\left(-\partial_{t} t\right)\right) \delta(t-h)=0
$$

in $\left(i_{h}\right)_{+} \mathcal{O}_{V}(* D)$, which contradicts the minimality of $b_{h}(s)$. 
Proof of the theorem. We consider, as in the last lemma, the $\mathcal{D}_{T \times V}$-module

$$
\left(i_{h}\right)_{+} \mathcal{O}_{V}(* D) e^{-f} \cong i_{*} \mathcal{O}_{V}(* D) e^{-f}\left[\partial_{t}\right]
$$

and the $\mathcal{D}_{V}\left[t \partial_{t}\right]$-submodule generated (over $\left.\mathcal{D}_{V}\left[t \partial_{t}\right]\right)$ by $\delta(t-h) e^{-f}$. The direct image $h_{+}\left(\mathcal{O}_{V}(* D) e^{-f}\right)$ is obtained in the standard way from $\left(i_{h}\right)_{+} \mathcal{O}_{V}(* D) e^{-f}$ as the relative de Rham complex of the projection $p_{1}: T \times V \rightarrow T$. In other words, we have

$$
\mathcal{H}^{i}\left(h_{+}\left(\mathcal{O}_{V}(* D) e^{-f}\right)\right)=\mathcal{H}^{i}\left(D R_{T \times V / T}^{n+\bullet}\left(\left(i_{h}\right)_{*} \mathcal{O}_{V}(* D) e^{-f}\left[\partial_{t}\right]\right)\right)
$$

Considering $\left(i_{h}\right)_{+} \mathcal{O}_{V}(* D) e^{-f}$ as a $\mathcal{D}_{V}$-module only, we thus have

$$
\mathcal{H}^{i}\left(h_{+}\left(\mathcal{O}_{V}(* D) e^{-f}\right)\right)=h_{*} \mathcal{H}^{i}\left(D R_{V}^{n+\bullet} \mathcal{O}_{V}(* D) e^{-f}\left[\partial_{t}\right]\right)
$$

Now it is well known (see, e.g., Mal75, proposition 2.1] or [Bjö93, proposition 2.2.10]), that for any left $\mathcal{D}_{V}$-module $\mathcal{L}$, the de Rham complex $D R^{\bullet}(\mathcal{L})$ is represented by the (shifted) derived tensor product $\Omega_{V}^{n} \stackrel{\mathbb{L}}{\otimes}_{\mathcal{D}_{V}} \mathcal{L}[-n]$, in particular, we have

$$
\mathcal{H}^{n}\left(D R_{V}^{\bullet}(\mathcal{L})\right) \cong \Omega_{V}^{n} \otimes_{\mathcal{D}_{V}} \mathcal{L}
$$

It follows that

$$
\mathcal{H}^{0}\left(h_{+}\left(\mathcal{O}_{V}(* D) e^{-f}\right)\right) \cong h_{*}\left(\Omega_{V}^{n} \otimes_{\mathcal{D}_{V}}\left(\mathcal{O}_{V}(* D) e^{-f}\left[\partial_{t}\right]\right)\right) .
$$

so that, taking global sections and considering again the isomorphism from lemma 7 , 4., we have

$$
H^{0}\left(V, \Omega_{V}^{n} \otimes_{\mathcal{D}_{V}}\left(\mathcal{O}_{V}(* D) e^{-f}\left[\partial_{t}\right]\right)\right) \cong G_{1}(* D)
$$

Notice that the section $\operatorname{vol} \otimes \delta(t-h) e^{-f}$ is mapped to the section $\omega_{1} / n=\mathrm{vol} / d h$ under this isomorphism. From the equation $\left(t \cdot h^{*}\left(\partial_{x_{i}}+a_{i}\right)-b_{h}\left(-\partial_{t} t\right)\right) \delta(t-h) e^{-f}=0$ in $\mathcal{O}_{V}(* D) e^{-f}\left[\partial_{t}\right]$ (equation (6)) we deduce that the element vol $\otimes\left(t \cdot h^{*}\left(\partial_{x_{i}}+a_{i}\right)-b_{h}\left(-\partial_{t} t\right)\right) \delta(t-h) e^{-f}$ is zero in $h_{*}\left(\Omega_{V}^{n} \otimes_{\mathcal{D}_{V}}\left(\mathcal{O}_{V}(* D) e^{-f}\left[\partial_{t}\right]\right)\right)$. Hence

$$
t \cdot\left(h^{*}\left(\partial_{x_{i}}+a_{i}\right)(\mathrm{vol})\right) \otimes \delta(t-h)^{-f}=b_{h}\left(-\partial_{t} t\right)\left(\operatorname{vol} \otimes \delta(t-h)^{-f}\right)
$$

holds in $h_{*}\left(\Omega_{V}^{n} \otimes_{\mathcal{D}_{V}}\left(\mathcal{O}_{V}(* D) e^{-f}\left[\partial_{t}\right]\right)\right)$, where the operator $h^{*}\left(\partial_{x_{i}}+a_{i}\right)$ acts on vol by the right $\mathcal{D}_{V^{-}}$ action on $\Omega_{V}^{n}$. Now develop the polynomial $h^{*}\left(y_{i}+a_{i}\right)$ as $h^{*}\left(y_{i}+a_{i}\right)=\sum_{1 \leq|I| \leq n} a_{I} y^{I}+h^{*}\left(a_{i}\right)$, then

$$
h^{*}\left(\partial_{x_{i}}+a_{i}\right)=\sum_{1 \leq|I| \leq n} a_{I} \partial_{x_{1}}^{i_{1}} \ldots \partial_{x_{n}}^{i_{n}}+h^{*}\left(a_{i}\right)
$$

and the action $h^{*}\left(\partial_{x_{i}}+a_{i}\right)(\mathrm{vol})$ is given by

$$
\sum_{1 \leq|I| \leq n} a_{I}(\underbrace{\operatorname{Lie}_{\partial_{x_{1}}} \cdots \operatorname{Lie}_{\partial_{x_{1}}}}_{i_{1}} \cdots \underbrace{\operatorname{Lie}_{\partial_{x_{n}}} \cdots \operatorname{Lie}_{\partial_{x_{n}}}}_{i_{n}})(\mathrm{vol})+h^{*}\left(a_{i}\right) \cdot \operatorname{vol}
$$

But obviously $\operatorname{Lie}_{\partial_{x_{i}}}$ vol $=0$ for any $i \in\{1, \ldots, n\}$, so that finally we see that the section vol $\otimes \delta(t-h) e^{-f}$ of $h_{*}\left(\Omega_{V}^{n} \otimes_{\mathcal{D}_{V}}\left(\mathcal{O}_{V}(* D) e^{-f}\left[\partial_{t}\right]\right)\right)$ is annihilated by $h^{*}\left(a_{i}\right) \cdot t-b_{h}\left(-\partial_{t} t\right)$. It follows that $b_{h}\left(-\partial_{t} t\right)$ sends $U_{0} G(* D)=V_{0} \mathbb{C}[t]\left\langle\partial_{t}\right\rangle \omega_{1}$ into $U_{-1} G(* D)$, hence, we have $b_{G_{1}(\log D)}(s+1) \mid b_{h}(s)$. Now the theorem follows as both $b_{h}$ and $b_{G_{1}(\log D)}$ are of degree $n$.

\section{Consequences and Examples}

Definition 15. Let $D$ be a reductive linear free divisor with defining equation $h \in \mathcal{O}_{V}$ and $f \in V^{*} a$ generic linear form. Consider, as in the last section, the logarithmic extension $G_{0}(\log D)$ of the family of Brieskorn lattices $G_{0}(* D)$ attached to $(f, h)$. We define the logarithmic Brieskorn lattice of $h$ to be the restriction $G_{0}(h):=i^{*}\left(G_{0}(\log D), \nabla\right)$, where $i: \mathbb{C} \times\{0\} \hookrightarrow \mathbb{C} \times T$. 
Notice that it follows from lemma 6 that $G_{0}(h)$ is independent of the choice of $f$ in $V^{*} \backslash D^{*}$, so that it makes sense to speak about the logarithmic Brieskorn lattice of $h$.

The next result, which is an easy consequence of theorem 13, can be considered as a variant of the corresponding classical statement of Malgrange (Mal75]) for the isolated singularity case.

Theorem 16. Let $\left(G_{0}(h), \nabla\right)$ be the logarithmic Brieskorn lattice of a reductive linear free divisor $D$. Then $\nabla$ is regular singular at $\theta=0$. Consider the saturation $\widetilde{G}_{0}(h):=\sum_{k>0}\left(\nabla_{\theta \partial_{\theta}}\right)^{k} G_{0}(h)$, which has a logarithmic pole sat $\theta=0$. Let $b_{\widetilde{G}_{0}(h)}(s)$ be the minimal polynomial of the residue endomorphism of $\nabla_{\theta}$ on $\widetilde{G}_{0}(h)$. Then $b_{\widetilde{G}_{0}(h)}(n s+1)=b_{h}(s)$.

Proof. The regularity follows easily from the particular form of the connection matrix (3). Namely, $G_{0}(h)$ is the Fourier-Laplace transformation of a regular $\mathbb{C}[r]\left\langle\partial_{r}\right\rangle$-module, hence, its regularity is equivalent to the nilpotency of the polar part of the connection matrix, which is obviously the case here, by putting $t=0$ in $A_{0}$. Now the saturation of $G_{0}(h)$ is easy to calculate: We put $\widetilde{\omega}_{i}:=\theta^{1-i} \omega_{i}$, then $G(\log D)=\oplus_{i=1}^{n} \mathbb{C}\left[\theta, \theta^{-1}, t\right] \widetilde{\omega}_{i}$, but $G_{0}(\log D) \subsetneq \oplus_{i=1}^{n} \mathbb{C}[\theta, t] \widetilde{\omega}_{i}$. It is evident that $\widetilde{G}_{0}(h)=\oplus_{i=1}^{n} \mathbb{C}[\theta] \widetilde{\omega}_{i}$, in particular, this module is invariant under $\theta \nabla_{\theta}$, i.e., logarithmic at $\theta=0$. We have $\left(\theta \partial_{\theta}\right) \underline{\widetilde{\omega}}=\underline{\widetilde{\omega}}$. $\left(\widetilde{A}_{0}+\operatorname{diag}\left(\left\{1-i-\nu_{i}\right\}_{i=1, \ldots, n}\right)\right.$. We see by theorem 13 that the residue eigenvalues of $\nabla_{\theta}$ at $\theta=0$ are the roots of the Bernstein polynomial of $h$ after dividing by $n$ and shift by -1 , and moreover that the residue endomorphism is regular (i.e., its minimal and characteristic polynomial coincide), as it has a cyclic generator. This proves the theorem.

Remark: One might ask what the meaning of the rescaling $n \cdot s$ occuring in $b_{\widetilde{G}_{0}(h)}(n s+1)$ is. The same kind of twist occurs in [MdGS08, proposition 4.5i(v)], where it is performed on the base, i.e., where the pull-back $u^{*}(G(* D))$ with $u: \mathbb{C}^{2} \rightarrow \mathbb{C} \times T,\left(\theta, t^{\prime}\right) \mapsto\left(\theta,\left(t^{\prime}\right)^{n}\right)$ is considered, and where it is shown that after this pull-back, the resulting bundle has the "rescaling property", i.e., that it is invariant under $\nabla_{\theta \partial_{\theta}-t^{\prime} \partial_{t^{\prime}}}$

The following easy consequence is a somewhat reverse argumentation compared to Malgrange's result, where the rationality of the roots of the Bernstein polynomial was deduced from the (known) quasiunipotency of the monodromy acting on the cohomology of the Milnor fibre of an isolated hypersurface singularity. In our case, the rationality of the roots of $b_{h}(s)$ is known (see, again, [GS08), but we deduce information on the (a priori unknown) monodromy of the logarithmic Brieskorn lattice $G_{0}(h)$. Moreover, we can use the results of GS08 to obtain a symmetry property of the spectrum at infinity of the logarithmic Brieskorn lattice, which was conjectured in MdGS08, corollary 5.6].

Corollary 17. The monodromy of the logarithmic Brieskorn lattice, i.e. of the local system associated to $G_{0}(h)\left[\theta^{-1}\right]:=G_{0}(h) \otimes_{\mathbb{C}[\theta]} \mathbb{C}\left[\theta, \theta^{-1}\right]$ is quasi-unipotent. Moreover, let $\alpha_{1}, \ldots, \alpha_{n}$ be the spectral numbers of $G_{0}(h)$ at infinity (i.e., the numbers $\nu_{i}$ from proposition 母), written as a non-decreasing sequence. Then $\alpha_{i}+\alpha_{n+1-i}=n-1$.

Proof. The eigenvalues of this monodromy are simply the exponentials of either the numbers $\nu_{i}$ or $\nu_{i}^{\prime}:=i-\nu_{i}$ from proposition 0 (or any other integer shift of them). The numbers $\nu_{i}^{\prime}$ are the roots of the Bernstein polynomial of $h$ shifted by one, as shown in theorem 13. These are known to be rational by Kas77. Similarly, if we denote the roots of $b_{h}$ by $\alpha_{1}^{\prime}, \ldots, \alpha_{n}^{\prime}$, with $\alpha_{i}^{\prime} \leq \alpha_{j}^{\prime}$ if $i \leq j$, then we know from [GS08, theorem 2.5.] that $\alpha_{i}^{\prime}+\alpha_{n+1-i}^{\prime}=-2$. From theorem 13 and proposition 10 we deduce that $\alpha_{j}=(j-1)-\alpha_{j}^{\prime}-1$ for any $j \in\{1, \ldots, n\}$, hence, $\alpha_{i}+\alpha_{n+1-i}=\left((i-1)-\alpha_{i}^{\prime}-1\right)+((n+1-i)-1-$ $\left.\alpha_{n+1-i}^{\prime}-1\right)=n-1$.

We outline another consequence of the theorem 13. Its interest is motivated by comparing the situation considered here with the one where $f$ is still a generic linear form, but $h$ is supposed to by an arbitrary monomial $h=\prod x_{i}^{w_{i}}$, i.e., non-reduced. The corresponding Gauß-Manin-systems resp. Brieskorn lattices have been studied in DS04, Dou08. It is known that they are closely related to the Mirror symmetry phenomenon, i.e., one constructs a Frobenius structure on the semi-universal unfolding of $f_{\mid h^{-1}(t)}, t \neq 0$ which is known to be isomorphic to the orbifold quantum cohomology of the weighted projective spaces. For a linear free divisor $D$, a similar construction of a Frobenius manifold has been carried out in MdGS08. Although these are not a priori mirrors of some variety or orbifold, the following corollary shows an interesting similarity with the case $h=\prod x_{i}^{w_{i}}$. 
Corollary 18. The spectrum at $\theta=\infty$ of both $\left(G_{0}(h), \nabla\right)$ and $(G(* D), \nabla)$ contains a (non-trivial) block of integer numbers $k, k+1, \ldots, n-1-k$ for some $k \in\{0, \ldots, n-1\}$.

Proof. For the spectrum of $\left(G_{0}(h), \nabla\right)$, this is obvious as this block corresponds to the root -1 of the Bernstein polynomial $b_{h}(s)$. For the spectrum of $(G(* D), \nabla)$, one shows the same statement by analyzing the construction of a good basis of $G(* D)$ from a good basis of $G_{0}(h)$ using algorithm 2 of MdGS08, lemma 4.11].

Notice that for the normal crossing case, the integer $k$ from above is equal to zero, i.e., the block mentioned above is the whole spectrum. This is not true in general, hence, the Frobenius structures constructed in MdGS08 are not, a priori, mirrors of quantum cohomology algebras of orbifolds, as zero is not, in general, an element of the spectrum. Still the analogy with the quantum orbifold cohomology, i.e., the fact that there is a block of increasing integer spectral numbers corresponding to the "untwisted sector" (see, e.g., [ri09, section 2.1.]) is rather intriguing.

Examples of Bernstein polynomials: We use the main result and the computations of spectral numbers in MdGS08 to obtain the roots of the Bernstein polynomials for the following reductive linear free divisors: Notice that the examples $E_{6}$ and "bracelet" are obtained by direct calculations in Singular

\begin{tabular}{c|c} 
(reductive) linear free divisor & Bernstein polynomial of $\boldsymbol{h}$ \\
\hline$A_{n}$ - quiver & $(s+1)^{n}$ \\
\hline$D_{m}$ - quiver & $\left(s+\frac{4}{3}\right)^{m-3} \cdot(s+1)^{2 m-4} \cdot\left(s+\frac{2}{3}\right)^{m-3}$ \\
\hline$E_{6}$ - quiver & $\left(s+\frac{7}{5}\right) \cdot\left(s+\frac{4}{3}\right)^{4} \cdot\left(s+\frac{6}{5}\right) \cdot(s+1)^{10} \cdot\left(s+\frac{4}{5}\right) \cdot\left(s+\frac{2}{3}\right)^{4} \cdot\left(s+\frac{3}{5}\right)$ \\
\hline$\star_{m}$ - quiver & $\prod_{l=0}^{m-3}\left(s+\frac{2(m-1)-l}{m}\right)^{l+1} \cdot(s+1)^{2(m-1)} \cdot \prod_{l=0}^{m-3}\left(s+\frac{m-1-l}{m}\right)^{m-l-2}$ \\
\hline bracelet & $\left(s+\frac{7}{6}\right) \cdot(s+1)^{2} \cdot\left(s+\frac{5}{6}\right)$
\end{tabular}

Table 1: Bernstein polynomials for some examples of linear free divisors

([GPS09]). On the other hand, the closed formulas for the star quiver and the $D$-series follows from rather involved combinatorial arguments, the details of which will appear in dGS09. Notice that the Bernstein polynomials for $D_{4}$ (which is equal to $\star_{3}$ ) and the bracelet are also calculated in [GS08. The one for $A_{n}$ is of course completely obvious and well known.

Let us finish this note with a remark and a conjecture exploiting further the analogy with the case of an isolated hypersurface singularity. We have seen that the theorem of Malgrange can be adapted for reductive linear free divisors using the logarithmic Brieskorn lattice from above. The regularity of $\left(G_{0}(h), \nabla\right)$ at $\theta=0$ suggest to study the spectrum in the classical sense of Varchenko (i.e., at $\theta=0$ ) of this lattice. We recall the definition and calculate two examples, in order to show that this spectrum contains additional information not present in roots of the Bernstein polynomial, similarly to the case of isolated singularities.

Definition 19. Let $(\mathbb{E}, \nabla)$ be a vector bundle on $\mathbb{C}=\operatorname{Spec} \mathbb{C}[\theta]$ equipped with a connection with a pole at zero of order two at most, which is regular singular. The localization $\mathbb{M}:=E \otimes_{\mathbb{C}[\theta]} \mathbb{C}\left[\theta, \theta^{-1}\right]$ has the structure of a holonomic $\mathbb{C}[\theta]\left\langle\partial_{\theta}\right\rangle$-module with a regular singularity at $\theta=0$. We suppose that the monodromy of its de Rham complex is quasi-unipotent. Denote by $V^{\bullet} \mathbb{M}$ the canonical $V$-filtration on $\mathbb{M}$, indexed by $\mathbb{Q}$, and which write as a decreasing filtration. Define the spectrum of $(E, \nabla)$ to be

$$
\operatorname{Sp}_{\theta=0}(E, \nabla):=\sum_{\alpha \in \mathbb{Q}} \frac{V^{\alpha} \mathbb{M} \cap \mathbb{E}}{V^{\alpha} \mathbb{M} \cap \theta \mathbb{E}+V^{>\alpha} \mathbb{M} \cap \mathbb{E}} \alpha \in \mathbb{Z}[\mathbb{Q}]
$$


where $V^{>\alpha} \mathbb{M}:=\cup_{\beta>\alpha} V^{\alpha} \mathbb{M}$.

As an example, we consider the case of the normal crossing divisor $D=\left\{h^{A_{n}}=\prod_{i=1}^{n} x_{i}=0\right\}$, which is the discriminant in the representation space of the quiver $A_{n}$. It was stated in [MdGS08] (but essentially well known before, due to the relation of this example to the quantum cohomology of the projective space $\left.\mathbb{P}^{n-1}\right)$ that we have $G_{0}\left(h^{A_{n}}\right):=\oplus_{i=1}^{n} \mathcal{O}_{\mathbb{C} \times\{0\}} \omega_{i}$, and

$$
\nabla(\underline{\omega})=\underline{\omega} \cdot\left[\frac{\widetilde{A}_{0}}{\theta}+\operatorname{diag}(0,1, \ldots, n-1)\right] \frac{d \theta}{\theta},
$$

$\widetilde{A}_{0}:=\left(A_{0}\right)_{\mid t=0}$. On the other hand, we take up the example of the star quiver with three exterior vertices studied in MdGS08, example 2.3(i)]. Notice that this is exactly the quiver $D_{4}$. Here $D \subset V=\mathbb{C}^{6}$, and $h^{\star 3}=h_{1}^{\star 3} \cdot h_{2}^{\star 3} \cdot h_{3}^{\star 3}$, where

$$
h_{1}^{\star_{3}}=\left|\begin{array}{cc}
a & b \\
d & e
\end{array}\right| \quad ; \quad h_{2}^{\star_{3}}=\left|\begin{array}{cc}
a & c \\
d & f
\end{array}\right| \quad ; \quad h_{3}^{\star_{3}}=\left|\begin{array}{cc}
b & c \\
e & f
\end{array}\right| .
$$

Following the various algorithms of loc.cit used to obtain good basis, we have that $G_{0}\left(h^{\star 3}\right):=\oplus_{i=1}^{6} \mathcal{O}_{\mathbb{C} \times\{0\}} \omega_{i}$, and

$$
\nabla(\underline{\omega})=\underline{\omega} \cdot\left[\frac{A_{0}}{\theta}+\operatorname{diag}(2,1,2,3,4,3)\right] \frac{d \theta}{\theta}
$$

Notice that this is the basis called $\underline{\omega}^{(2)}$ in loc.cit.

Proposition 20. 1. The spectrum at $\theta=0$ for $h^{A_{n}}$ is

$$
\operatorname{Sp}_{\theta=0}\left(G_{0}\left(h^{A_{n}}\right), \nabla\right)=(0,1, \ldots, n-1) \in \mathbb{Z}[\mathbb{Q}],
$$

hence, it is equal to the spectrum at $\theta=\infty$ of both $\left(G_{0}(h), \nabla\right)$ and $(G(* D), \nabla)$ (so that in this case we do not get more informations from the spectrum at $\theta=0$ than those contained in the roots of $\left.b_{h}(s)\right)$.

2. The spectrum at $\theta=0$ for $h^{\star 3}$ is given by

$$
\operatorname{Sp}_{\theta=0}\left(G_{0}\left(h^{\star 3}\right), \nabla\right)=(-2,1,2,3,4,7) \in \mathbb{Z}[\mathbb{Q}],
$$

hence, different from $\mathrm{Sp}_{\theta=0}\left(G_{0}(h), \nabla\right)$ and not directly related to $b_{h}(s)=\left(s+\frac{4}{3}\right)(s+1)^{4}\left(s+\frac{2}{3}\right)$.

Proof. 1. One can calculate directly that $G_{0}\left(h^{A_{n}}\right)$ can be generated by elementary sections, which implies that $\operatorname{Sp}_{\theta=0}\left(G_{0}\left(h^{A_{n}}\right), \nabla\right)$ is equal to the spectrum at $\theta=\infty$, i.e., $\operatorname{Sp}_{\theta=0}\left(G_{0}\left(h^{A_{n}}\right), \nabla\right)=$ $(0,1, \ldots, n-1)$. However, this can also be obtained in a more abstract way: For any linear free divisor $D$, the analytic object corresponding to the restriction of $G_{0}(* D)$ to $\mathbb{C} \times(T \backslash\{0\})$ is known (after a finite ramification of order $n$ ) to be a Sabbah orbit of TERP-structures (see the remark after the proof of theorem 16 and MdGS08, proposition $4.5(\mathrm{v})])$. In the $A_{n}$-case, it is easy to see that the extension $G_{0}(\log D)$ is exactly the extension ${ }_{0} \mathcal{E}$ considered in HS07, proof of theorem 7.3 and lemma 6.11] and the logarithmic Brieskorn lattice $G_{0}(h)$ is isomorphic to the limit $\mathcal{G}_{0}$ considered in loc.cit, proof of theorem 7.3 and lemma 6.12. It was shown in the proof of theorem 7.3 of loc.cit. that $\mathcal{G}_{0}$ is generated by elementary sections.

2. In the $\star_{3}$-case, one cannot apply the previous reasoning. Hence a direct calculation is necessary. We explain parts of it, leaving the details to the reader. From the connection matrix given above we see that $\left(\theta \partial_{\theta}\right) \omega_{6}=3 \omega_{6}$, and $\left(\theta \partial_{\theta}\right) \omega_{5}=4 \omega_{5}-\theta^{-1} \omega_{6}$. We make the Ansatz

$$
\omega_{5}=\alpha \theta^{-1} \omega_{6}+s_{4}
$$

where $s_{4}$ is a section of $G_{0}(h)\left[\theta^{-1}\right]$ satisfying $\left(\theta \partial_{\theta}\right)\left(s_{4}\right)=4 \cdot s_{4}$. We obtain

$$
\left(\theta \partial_{\theta}\right) \omega_{5}=2 \alpha \theta^{-1} \omega_{6}+4 s_{4} \stackrel{!}{=}(4 \alpha-1) \theta^{-1} \omega_{6}+4 s_{4}
$$


from which we conclude that $\omega_{5}=\frac{1}{2} \theta^{-1} \omega_{6}+s_{4}$. Similarly, the equation $\left(\theta \partial_{\theta}\right) \omega_{4}=3 \omega_{4}-\theta^{-1} \omega_{5}$ is satisfied by putting

$$
\omega_{4}=\frac{1}{8} \beta_{1} \cdot \theta^{-2} \omega_{6}+s_{3}
$$

where $s_{3} \in G_{0}(h)\left[\theta^{-1}\right]$ is a section satisfying $\left(\theta \partial_{\theta}\right) s_{3}=3 s_{3}+\theta^{-1} s_{4}$. Continuing this way we see that the elements of our basis $\underline{\omega}$ can be written as finite sums of elementary sections in the following way:

$$
\begin{gathered}
\omega_{1}=\frac{1}{128} \theta^{-5} \omega_{6}+\frac{1}{16} \theta^{-4} s_{4}+\frac{1}{8} \theta^{-3} s_{3}+\frac{1}{4} \theta^{-2} s_{2}+\frac{1}{2} \theta^{-1} s_{1}+\widetilde{s}_{2} \\
\omega_{2}=\frac{1}{32} \theta^{-4} \omega_{6}+s_{1} ; \omega_{3}=\frac{1}{16} \theta^{-3} \omega_{6}-s_{2} ; \omega_{4}=\frac{1}{8} \theta^{-2} \omega_{6}+s_{3} \\
\omega_{5}=\frac{1}{2} \theta^{-1} \omega_{6}+s_{4} ; \omega_{6}=\omega_{6}
\end{gathered}
$$

where $s_{1}, s_{2}, s_{3}, s_{4}, \widetilde{s}_{2}$ are sections of $G_{0}(h)\left[\theta^{-1}\right]$ satisfying

$$
\begin{aligned}
& \left(\theta \partial_{\theta}\right) s_{1}=s_{1}+\theta^{-1} s_{2} ;\left(\theta \partial_{\theta}\right) s_{2}=2 s_{2}+\theta^{-1} s_{3} \\
& \left(\theta \partial_{\theta}\right) s_{3}=3 s_{3}+\theta^{-1} s_{4} ;\left(\theta \partial_{\theta}\right) s_{4}=4 s_{4} \\
& \left(\theta \partial_{\theta}\right) \widetilde{s}_{2}=2 \widetilde{s}_{4}
\end{aligned}
$$

Now it is easy to calculate an upper triangular base change yielding a good basis and to show that the spectrum is

$$
\operatorname{Sp}_{\theta=0}\left(G_{0}\left(h^{\star 3}\right), \nabla\right)=(-2,1,2,3,4,7) \in \mathbb{Z}[\mathbb{Q}]
$$

as required.

Based on the computations of these examples, we state the following conjecture, which is related to corollary 17 as well as to MdGS08, conjecture 5.5.].

Conjecture 21. Let $h$ be the defining equation of a reductive linear free divisor $D \subset V=\mathbb{C}^{n}$. Then the spectrum of its logarithmic Brieskorn lattice $\left(G_{0}(h), \nabla\right)$ at $\theta=0$ is symmetric around $\frac{n-1}{2}$.

Remark: There are several questions one may ask about the spectrum at $\theta=0$. First, it is surprising that negative numbers (even smaller than -1 ) occur in this spectrum. One might want to understand the possibly range for the spectrum, as well as the difference to the roots of $b_{h}$, when multiplied by $n$. This should be compared to the results in HS99] for isolated singularities, in particular, lemma 3.4 of loc.cit.

\section{References}

[Ber72] I. N. Bernstein, Analytic continuation of generalized functions with respect to a parameter, Functional Analysis and Its Applications 6 (1972), no. 4, 26-40.

[Bjö93] Jan-Erik Björk, Analytic D-modules and applications, Mathematics and its Applications, vol. 247, Kluwer Academic Publishers Group, Dordrecht, 1993.

[BM06] Ragnar-Olaf Buchweitz and David Mond, Linear free divisors and quiver representations, Singularities and computer algebra (Cambridge) (Christoph Lossen and Gerhard Pfister, eds.), London Math. Soc. Lecture Note Ser., vol. 324, Cambridge Univ. Press, 2006, Papers from the conference held at the University of Kaiserslautern, Kaiserslautern, October 18-20, 2004, pp. 41-77.

[dGS09] Ignacio de Gregorio and Christian Sevenheck, Good bases for some linear free divisors associated to quiver representations, work in progress, 2009.

[Dou08] Antoine Douai, Examples of limits of Frobenius (type) structures: The singularity case, Preprint math.AG/0806.2011, 2008. 
[DS03] Antoine Douai and Claude Sabbah, Gauss-Manin systems, Brieskorn lattices and Frobenius structures. I, Ann. Inst. Fourier (Grenoble) 53 (2003), no. 4, 1055-1116.

[DS04] - Gauss-Manin systems, Brieskorn lattices and Frobenius structures. II, Frobenius manifolds, Aspects Math., E36, Vieweg, Wiesbaden, 2004, pp. 1-18.

[GMNS09] Michel Granger, David Mond, Alicia Nieto, and Mathias Schulze, Linear free divisors and the global logarithmic comparison theorem., Ann. Inst. Fourier (Grenoble) 59 (2009), no. 1, 811-850.

[GPS09] G.-M. Greuel, G. Pfister, and H. Schönemann, Singular 3.1.0 - A computer algebra system for polynomial computations, http://www.singular.uni-kl.de.

[GS08] Michel Granger and Mathias Schulze, On the symmetry of b-functions of linear free divisors., Preprint math.AG/0807.0560, 2008.

[Gyo91] Akihiko Gyoja, Theory of prehomogeneous vector spaces without regularity condition, Publ. Res. Inst. Math. Sci. 27 (1991), no. 6, 861-922. MR MR1145669 (93f:22018)

[HS99] Claus Hertling and Colin Stahlke, Bernstein polynomial and Tjurina number, Geom. Dedicata 75 (1999), no. 2, 137-176.

[HS07] Claus Hertling and Christian Sevenheck, Nilpotent orbits of a generalization of Hodge structures., J. Reine Angew. Math. 609 (2007), 23-80.

[Iri09] Hiroshi Iritani, An integral structure in quantum cohomology and mirror symmetry for toric orbifolds, Preprint math.AG/0903.1463, 2009.

[Kas77] Masaki Kashiwara, B-functions and holonomic systems. Rationality of roots of B-functions, Invent. Math. 38 (1976/77), no. 1, 33-53.

[Mal75] Bernard Malgrange, Le polynôme de Bernstein d'une singularité isolée, Fourier integral operators and partial differential equations (Colloq. Internat., Univ. Nice, Nice, 1974) (J. Chazarain, ed.), Lecture Notes in Mathematics, Vol. 459, Springer, Berlin, 1975, Colloque International, réuni à l'Université de Nice, Nice, du 20 au 25 mai 1974, pp. 98-119. Lecture Notes in Math., Vol. 459.

[MdGS08] David Mond, Ignacio de Gregorio, and Christian Sevenheck, Linear free divisors and Frobenius manifolds., Preprint math.AG/0802.4188, to appear in "Compositio Mathematica", 2008.

[Meb04] Zoghman Mebkhout, Le théorème de positivité, le théorème de comparaison et le théorème d'existence de Riemann, in Maisonobe and Narváez Macarro MNM04, Papers from the CIMPA Summer School held in Séville, September 2-13, 1996, pp. 165-310.

[MM04] Philippe Maisonobe and Zoghman Mebkhout, Le théorème de comparaison pour les cycles évanescents, in Maisonobe and Narváez Macarro MNM04, Papers from the CIMPA Summer School held in Séville, September 2-13, 1996, pp. 311-389.

[MNM04] Philippe Maisonobe and Luis Narváez Macarro (eds.), Éléments de la théorie des systèmes différentiels géométriques, Séminaires et Congrès [Seminars and Congresses], vol. 8, Société Mathématique de France, Paris, 2004, Papers from the CIMPA Summer School held in Séville, September 2-13, 1996.

[Rou06a] Céline Roucairol, Irregularity of an analogue of the Gauss-Manin systems, Bull. Soc. Math. France 134 (2006), no. 2, 269-286.

[Rou06b] - The irregularity of the direct image of some $\mathcal{D}$-modules, Publ. Res. Inst. Math. Sci. 42 (2006), no. 4, 923-932.

[Rou07] - Formal structure of direct image of holonomic $\mathcal{D}$-modules of exponential type, Manuscripta Math. 124 (2007), no. 3, 299-318. 
[Sai80] Kyoji Saito, Theory of logarithmic differential forms and logarithmic vector fields, J. Fac. Sci. Univ. Tokyo Sect. IA Math. 27 (1980), no. 2, 265-291.

[SK77] M. Sato and T. Kimura, A classification of irreducible prehomogeneous vector spaces and their relative invariants, Nagoya Math. J. 65 (1977), 1-155.

Lehrstuhl für Mathematik VI

Institut für Mathematik

Universität Mannheim, A 5, 6

68131 Mannheim

Germany

Christian.Sevenheck@math.uni-mannheim.de 